\title{
Insurance Status and Hospital Care for Myocardial Infarction, Stroke, and Pneumonia
}

Omar Hasan, MBBS, MPH $^{1,2}$ E. John Orav, PhD ${ }^{1}$ LeRoi S. Hicks, MD, MPH ${ }^{1,2,3}$

\author{
${ }^{1}$ Department of Medicine, Division of General Medicine, Brigham and Women's Hospital, Boston, Massachusetts. \\ ${ }^{2}$ Department of Medicine, Brigham and Women's-Faulkner Hospitalist Service, Brigham and Women's Hospital, \\ Boston, Massachusetts. \\ ${ }^{3}$ Department of Health Care Policy, Harvard Medical School, Boston, Massachusetts.
}

Disclosure: Nothing to report.

BACKGROUND: Despite widely documented variations in health care outcomes by insurance status, few nationally representative studies have examined such disparities in the inpatient setting.

OBJECTIVE: To determine whether there are insurance-related differences in hospital care for 3 common medical conditions. DESIGN AND SUBJECTS: Retrospective database analysis of 154,381 adult discharges (age 18-64 years) with a principal diagnosis of acute myocardial infarction (AMI), stroke, or pneumonia from the 2005 Nationwide Inpatient Sample (NIS). MEASUREMENTS: For each diagnosis, we compared in-hospital mortality, length of stay (LOS), and cost per hospitalization for Medicaid and uninsured patients with the privately insured.

RESULTS: Compared with the privately insured, in-hospital mortality among AMI and stroke patients was significantly higher for the uninsured (adjusted odds ratio [OR] 1.52, 95\% confidence interval [CI] [1.24-1.85] for AMI and 1.49 [1.29-1.72] for stroke) and among pneumonia patients was significantly higher for Medicaid recipients (1.21 [1.01-1.45]). Excluding patients who died during hospitalization, LOS was consistently longer for Medicaid recipients for all 3 conditions (adjusted ratio 1.07, 95\% CI [1.05-1.09] for AMI, 1.17 [1.14-1.20] for stroke, and 1.04 [1.03-1.06] for pneumonia), although costs were significantly higher for Medicaid recipients for only 2 of the 3 conditions (adjusted ratio 1.06, 95\% CI [1.04-1.09] for stroke and 1.05 [1.04-1.07] for pneumonia).

CONCLUSIONS: In this nationally representative study of working-age Americans hospitalized for 3 common medical conditions, significantly lower in-hospital mortality was noted for privately insured patients compared with the uninsured or Medicaid recipients. Interventions to reduce insurance-related gaps in inpatient quality of care should be investigated.

Journal of Hospital Medicine 2010;5:452-459. ㅇ 2010 Society of Hospital Medicine.

\section{KEYWORDS: hospital cost, in-hospital mortality, insurance status, length of stay, uninsured.}

Additional Supporting Information may be found in the online version of this article.

With about 1 in 5 working-age Americans (age 18-64 years) currently uninsured and a large number relying on Medicaid, adequate access to quality health care services is becoming increasingly difficult. ${ }^{1}$ Substantial literature has accumulated over the years suggesting that access and quality in health care are closely linked to an individual's health insurance status. ${ }^{2-11}$ Some studies indicate that being uninsured or publicly insured is associated with negative health consequences. $^{12,13}$ Although the Medicaid program has improved access for qualifying low-income individuals, significant gaps in access and quality remain., ${ }^{2,5,11,14-19}$ These issues are likely to become more pervasive should there be further modifications to state Medicaid funding in response to the unfolding economic crisis.

Although numerous studies have focused on insurancerelated disparities in the outpatient setting, few nationally representative studies have examined such disparities among hospitalized patients. A cross-sectional study of a large hospital database from 1987 reported higher risk- adjusted in-hospital mortality, shorter length of stay (LOS), and lower procedure use among uninsured patients. ${ }^{9}$ A more recent analysis, limited to patients admitted with stroke, reported significant variation in hospital care associated with insurance status. ${ }^{15}$ Other studies reporting myocardial infarction registry and quality improvement program data are biased by the self-selection of large urban teaching hospitals. ${ }^{16-18}$ To our knowledge, no nationally representative study has focused on the impact of insurance coverage on hospital care for common medical conditions among working-age Americans, the fastest growing segment of the uninsured population.

To address this gap in knowledge, we analyzed a nationally representative hospital database to determine whether there are significant insurance-related disparities in in-hospital mortality, LOS, and cost per hospitalization for 3 common medical conditions among working-age adults, and, if present, to determine whether these disparities are due to differences in disease severity and comorbidities, and whether 
these disparities are affected by the proportion of uninsured and Medicaid patients receiving care in each hospital.

\section{Methods}

\section{Design and Subjects}

We examined data from the 2005 Nationwide Inpatient Sample (NIS), a nationally representative database of hospital inpatient stays maintained by the Agency for Healthcare Research and Quality (AHRQ) as part of the Healthcare Cost and Utilization Project (HCUP). ${ }^{20,21}$ The NIS is a stratified probability sample of $20 \%$ of all US community hospitals, including public hospitals, academic medical centers, and specialty hospitals. Long-term care hospitals, psychiatric hospitals, and alcoholism/chemical-dependency treatment facilities are excluded. The 2005 NIS contains data on 7,995,048 discharges from 1054 hospitals located in 37 States and is designed to be representative of all acute care discharges from all US community hospitals. ${ }^{21}$

We identified discharges with a principal diagnosis of acute myocardial infarction (AMI), stroke, and pneumonia using International Classification of Diseases, 9th Revision, Clinical Modification (ICD-9-CM) codes specified in the AHRQ definitions of Inpatient Quality Indicators (Supporting Information Appendix). ${ }^{22}$ These 3 conditions are among the leading causes of noncancer inpatient deaths in patients under 65 years old, ${ }^{23}$ and evidence suggests that high mortality may be associated with deficiencies in the quality of inpatient care. ${ }^{24}$

We confined our analysis to patients 18 to 64 years of age, since this population is most at risk of being uninsured or underinsured. ${ }^{25}$ We excluded pregnant women because they account for an unusually high proportion of uninsured discharges and were relatively few in our cohort. ${ }^{26}$ In addition, we excluded patients transferred to another acute care hospital and patients missing payer source and discharge disposition. Our study protocol was approved by the Partners Human Research Committee.

\section{Study Variables}

We categorized insurance status as privately insured, uninsured, Medicaid, or Medicare. We defined "privately insured" patients as those having either Blue Cross or another commercial carrier listed as the primary payer and "uninsured" patients as those having either no charge or self-pay listed as the primary payer. $^{27}$ Other governmental payer categories were noted to share several characteristics with Medicare patients and comprised only a small proportion of the sample, and were thus included with Medicare. In order to account for NIS's sampling scheme and accurately apply sample weights in our analysis, we used Medicare as a separate category. However, since Medicare patients age 18 to 64 years represent a fundamentally different population that is primarily disabled or very ill, only results of privately insured, uninsured, and Medicaid patients are reported.

We selected in-hospital mortality as the outcome measure and LOS and cost per hospitalization as measures of resource utilization. The NIS includes a binary indicator variable for in-hospital mortality and specifies inpatient LOS in integers, with same-day stays coded as 0 . NIS's cost estimates are based on hospital cost reports submitted to the Centers for Medicare and Medicaid Services. To test the validity of our cost analyses, we performed parallel analyses using hospital charges as a measure of utilization (charges include hospital overhead, charity care, and bad debt). The resulting adjusted ratios differed little from cost ratios and we opted to report only the details of our cost analyses.

In order to assess the independent association between insurance status and the outcome measures listed above, we selected covariates for inclusion in multivariable models based on the existing literature. Patient covariates included: age group (18-34 years, 35-49 years, 50-64 years), sex (male/ female), race/ethnicity (non-Hispanic white, non-Hispanic black, Hispanic, other, missing), median income by zip code of residence (less than $\$ 37,000, \$ 37,000-\$ 45,999, \$ 46,000$ $\$ 60,999, \$ 61,000$ or more), admission through the emergency department (yes/no), admission on a weekend (yes/ no), measures of disease severity, and comorbidity indicators. Measures of disease severity specific to each outcome are assigned in the NIS using criteria developed by Medstat (Medstat Disease Staging Software Version 5.2, Thomson Medstat Inc., Ann Arbor, MI). Severity is categorized into 5 levels, with a higher level indicating greater risk. We recorded comorbidities for each patient in our sample using HCUP Comorbidity Software, Version 3.2 (www.hcupus.ahrq.gov/tools_software.jsp) based on comorbidity measures reported by Elixhauser et al. ${ }^{28}$

Hospital covariates included: bed size (small, medium, large), ownership/control (private, government, private or government), geographic region (northeast, midwest, south, west), teaching status (teaching, non-teaching), and the proportion of uninsured and Medicaid patients (combined) admitted to each hospital for AMI, stroke, or pneumonia. The actual number of hospital beds in each bed size category varies according to a hospital's geographic region and teaching status. ${ }^{27}$ Ownership/control, geographic region, and teaching status are assigned according to information from the American Hospital Association Annual Survey of Hospitals. The proportion of uninsured and Medicaid patients admitted to each hospital was found to have a nonmonotonic relationship with the outcomes being assessed and was thus treated as a 6-level categorical variable with the following levels: $0 \%$ to $10 \%, 11 \%$ to $20 \%, 21 \%$ to $30 \%$, $31 \%$ to $40 \%, 41 \%$ to $50 \%$, and $51 \%$ to $100 \%$.

\section{Statistical Analysis}

Summary statistics were constructed at the patient level and differences in proportions were evaluated with the chisquare test. We employed direct standardization, using the age and sex distribution of the entire cohort, to compute age-standardized and sex-standardized estimates for each insurance group and compared them using the chi-square

2010 Society of Hospital Medicine DOI 10.1002/jhm.687 View this article online at wileyonlinelibrary.com. 
test for in-hospital mortality and $t$ test for log transformed LOS and cost per hospitalization. For each condition, we developed multivariable logistic regression models for inhospital mortality and multivariable linear regression models for log transformed LOS and cost. The patient was the unit of analysis in all models.

In order to elucidate the contribution of disease severity and comorbidities and the proportion of uninsured and Medicaid patients admitted to each hospital, we fitted 3 sequential models for each outcome measure: Model 1 adjusted for patient sociodemographic characteristics and hospital characteristics with the exception of the covariate for the proportion of uninsured and Medicaid patients, Model 2 adjusted for all covariates in the preceding model as well as patients' comorbidities and severity of principal diagnosis, and Model 3 adjusted for all previously mentioned covariates as well as the proportion of uninsured and Medicaid patients admitted to each hospital. We excluded patients who died during hospitalization from the models for LOS and cost. We exponentiated the effect estimates from the log transformed linear regression models so that the adjusted ratio represents the percentage change in the mean LOS or mean cost.

To determine whether the association between patients' insurance status and in-hospital mortality was modified by seeking care in hospitals treating a smaller or larger proportion of uninsured and Medicaid patients, we entered an interaction term for insurance status and proportion of uninsured and Medicaid patients in the final models (Model 3) for our primary outcome of in-hospital mortality. However, since no significant interaction was found for any of the 3 conditions, this term was removed from the models and results from the interaction models are not described. In order to assess model specification for the linear regression models, we evaluated the normality of model residuals and found that these were approximately normally distributed. Lastly, we attempted to test the robustness of our analyses by creating fixed effects models that controlled for hospital site but were unable to do so due to the computational limitations of available software packages that could not render fixed effects models with more than 1000 hospital sites.

For all variables except race/ethnicity, data were missing for less than $3 \%$ of patients, so we excluded these individuals from adjusted analyses. However, race/ethnicity data were missing for $29 \%$ of the sample and were analyzed in 3 different ways, namely, with the missing data treated as a separate covariate level, with the missing data removed from the analysis, and with the missing data assigned to the majority covariate level (white race). The results of our analysis were unchanged no matter how the missing values were assigned. As a result, missing values for race/ethnicity were treated as a separate covariate level in the final analysis. ${ }^{15}$ Sociodemographic characteristics of patients with missing race/ethnicity information were similar to those with complete data.
We used SUDAAN (Release 9.0.1, Research Triangle Institute, NC) to account for NIS's sampling scheme and generalized estimating equations to adjust for the clustering of patients within hospitals and hospitals within sampling strata. $^{29}$ In order to account for NIS's stratified probability sampling scheme, SUDAAN uses Taylor series linearization for robust variance estimation of descriptive statistics and regression parameters. ${ }^{30,31}$ We present 2-tailed $P$ values or $95 \%$ confidence intervals (CIs) for all statistical comparisons.

\section{Results}

\section{Patient and Hospital Characteristics}

The final cohort comprised of 154,381 patients discharged from 1018 hospitals in 37 states during calendar year 2005 (Table 1). This cohort was representative of 755,346 working-age Americans, representing approximately 225,947 cases of AMI (29.9\%), 151,812 cases of stroke $(20.1 \%)$, and 377,588 cases of pneumonia $(50.0 \%)$. Of these patients, $47.5 \%$ were privately insured, $12.0 \%$ were uninsured, $17.0 \%$ received Medicaid, and $23.5 \%$ were assigned to Medicare. Compared with the privately insured, uninsured and Medicaid patients were generally younger, less likely to be white, more likely to have lower income, and more likely to be admitted through the emergency department. Of the 1018 hospitals included in our study, close to half $(44.3 \%)$ were small, with bedsize ranging from 24 to 249. A large number of hospitals were located in the South (39.9\%), and $14.9 \%$ were designated teaching hospitals.

Compared with privately insured patients, a larger proportion of uninsured and Medicaid patients had higher predicted mortality levels (Table 2). Medicaid patients had a disproportionately higher predicted LOS, whereas predicted resource demand was higher among privately insured patients. Hypertension (48\%), chronic pulmonary disease (29.5\%), and uncomplicated diabetes $(21.5 \%)$ were the 3 most common comorbidities in the study cohort, with a generally higher prevalence of comorbidities among Medicaid patients.

\section{In-Hospital Mortality}

Compared with the privately insured, age-standardized and sex-standardized in-hospital mortality for AMI and stroke was significantly higher for uninsured and Medicaid patients (Table 3). Among pneumonia patients, Medicaid recipients had significantly higher in-hospital mortality compared with privately insured and uninsured patients.

After multivariable adjustment for additional patient and hospital characteristics, uninsured AMI and stroke patients continued to have significantly higher in-hospital mortality compared with the privately insured (Table 4). Among pneumonia patients, Medicaid recipients persisted in having significantly higher in-hospital mortality than the privately insured. 


\begin{tabular}{|c|c|c|c|}
\hline Characteristic* & $\begin{array}{l}\text { Privately insured } \\
(\mathrm{n}=73,256)\end{array}$ & $\begin{array}{l}\text { Uninsured } \\
(n=18,531)\end{array}$ & $\begin{array}{l}\text { Medicaid } \\
(\mathrm{n}=26,222)\end{array}$ \\
\hline \multicolumn{4}{|l|}{ Principal diagnosis (\%) } \\
\hline Acute myocardial infarction & 36.7 & 31.2 & 19.7 \\
\hline Stroke & 20.6 & 23.7 & 19.9 \\
\hline Pneumonia & 42.7 & 45.2 & 60.4 \\
\hline \multicolumn{4}{|l|}{ Age group (\%) } \\
\hline 18-34 years & 6.8 & 13.0 & 13.7 \\
\hline $35-49$ years & 27.6 & 36.9 & 33.2 \\
\hline 50-64 years & 65.7 & 50.1 & 53.2 \\
\hline Male sex $(\%)$ & 59.3 & 62.3 & 46.6 \\
\hline \multicolumn{4}{|l|}{ Race or ethnicity (\%) } \\
\hline White & 55.7 & 41.5 & 38.0 \\
\hline Black & 7.6 & 14.8 & 16.6 \\
\hline Hispanic & 4.8 & 10.5 & 10.4 \\
\hline Other race & 3.6 & 4.7 & 5.2 \\
\hline Missing & 28.4 & 29.0 & 29.7 \\
\hline \multicolumn{4}{|l|}{ Median income by ZIP code (\%) } \\
\hline$<\$ 37,000$ & 21.5 & 36.7 & 43.0 \\
\hline$\$ 37,000-\$ 45,999$ & 25.2 & 27.8 & 27.1 \\
\hline$\$ 46,000-\$ 60,999$ & 26.3 & 20.3 & 17.6 \\
\hline$\geq \$ 61,000$ & 24.8 & 11.5 & 8.4 \\
\hline Emergency admission (\%) & 63.3 & 75.6 & 72.9 \\
\hline Weekend admission (\%) & 24.5 & 26.2 & 25.1 \\
\hline \multicolumn{4}{|l|}{ Hospital bed size (\%) } \\
\hline Small & 8.9 & 10.3 & 11.4 \\
\hline Medium & 24.0 & 22.3 & 25.9 \\
\hline Large & 67.1 & 67.5 & 62.8 \\
\hline \multicolumn{4}{|l|}{ Hospital control (\%) } \\
\hline Private & 33.8 & 34.8 & 34.4 \\
\hline Government (nonfederal) & 6.7 & 9.7 & 8.3 \\
\hline Private or government & 59.5 & 55.5 & 57.3 \\
\hline \multicolumn{4}{|l|}{ Hospital region (\%) } \\
\hline Northeast & 17.4 & 12.5 & 17.6 \\
\hline Midwest & 25.7 & 19.4 & 20.9 \\
\hline South & 39.5 & 56.8 & 42.4 \\
\hline West & 17.4 & 11.3 & 19.2 \\
\hline Teaching hospital (\%) & 41.7 & 43.8 & 43.3 \\
\hline
\end{tabular}

\section{LOS}

Among AMI and stroke patients, age-standardized and sexstandardized mean LOS was significantly longer for the uninsured and Medicaid recipients compared with the privately insured (Table 3). Among pneumonia patients, the uninsured had a slightly shorter mean LOS compared with the privately insured whereas Medicaid recipients averaged the longest LOS.

These insurance-related disparities in LOS among pneumonia patients persisted after multivariable adjustment (Table 4). Among AMI patients, only Medicaid recipients persisted in having a significantly longer LOS than the privately insured. Among stroke patients, both the uninsured and Medicaid recipients averaged a longer LOS compared with the privately insured.

\begin{tabular}{|c|c|c|c|}
\hline Characteristic* & $\begin{array}{l}\text { Privately insured } \\
(\mathrm{n}=73,256)\end{array}$ & $\begin{array}{l}\text { Uninsured } \\
(\mathrm{n}=18,531)\end{array}$ & $\begin{array}{l}\text { Medicaid } \\
(n=26,222)\end{array}$ \\
\hline \multicolumn{4}{|l|}{ Medstat disease staging ${ }^{\dagger}(\%)$} \\
\hline Mortality level 1 & 50.8 & 45.4 & 36.7 \\
\hline Mortality level 2 & 44.0 & 49.1 & 56.7 \\
\hline Mortality level 3 & 5.3 & 5.5 & 6.7 \\
\hline Length of stay level 1 & 66.8 & 71.6 & 53.8 \\
\hline Length of stay level 2 & 28.5 & 24.5 & 39.3 \\
\hline Length of stay level 3 & 4.8 & 3.8 & 6.9 \\
\hline Resource demand level 1 & 45.2 & 54.2 & 48.5 \\
\hline Resource demand level 2 & 40.5 & 34.2 & 39.2 \\
\hline Resource demand level 3 & 14.2 & 11.7 & 12.3 \\
\hline \multicolumn{4}{|l|}{ Coexisting medical conditions (\%) } \\
\hline Congestive heart failure & 4.7 & 4.8 & 10.1 \\
\hline Valvular disease & 2.8 & 2.0 & 2.7 \\
\hline Pulmonary circulation disease & 0.8 & 0.6 & 1.5 \\
\hline Peripheral vascular disease & 3.2 & 2.2 & 3.2 \\
\hline Paralysis & 1.2 & 0.8 & 3.5 \\
\hline Other neurological disorders & 2.4 & 1.9 & 7.3 \\
\hline Chronic pulmonary disease & 23.6 & 22.4 & 37.7 \\
\hline Uncomplicated diabetes & 19.6 & 18.6 & 23.4 \\
\hline Complicated diabetes & 3.3 & 2.1 & 4.9 \\
\hline Hypothyroidism & 5.6 & 2.7 & 4.7 \\
\hline Renal failure & 3.0 & 1.9 & 5.6 \\
\hline Liver disease & 1.6 & 2.5 & 4.4 \\
\hline Peptic ulcer disease & $<0.5$ & $<0.5$ & $<0.5$ \\
\hline AIDS & 0.1 & 0.1 & 0.4 \\
\hline Lymphoma & 1.1 & 0.4 & 0.6 \\
\hline Metastatic cancer & 2.1 & 0.7 & 2.2 \\
\hline Non-metastatic solid tumor & 1.5 & 0.8 & 2.1 \\
\hline Collagen vascular diseases & 2.3 & 0.9 & 2.3 \\
\hline Coagulopathy & 2.7 & 2.4 & 3.4 \\
\hline Obesity & 10.3 & 8.2 & 9.3 \\
\hline Weight loss & 1.6 & 1.8 & 3.3 \\
\hline Fluid and electrolyte disorders & 18.3 & 19.4 & 23.8 \\
\hline Chronic blood loss anemia & 0.6 & 0.6 & 0.8 \\
\hline Deficiency anemias & 8.6 & 8.5 & 13.4 \\
\hline Alcohol abuse & 3.3 & 9.8 & 8.3 \\
\hline Drug abuse & 1.9 & 10.2 & 9.8 \\
\hline Psychoses & 1.5 & 1.9 & 6.8 \\
\hline Depression & 7.2 & 4.8 & 9.9 \\
\hline Hypertension & 48.0 & 44.1 & 45.7 \\
\hline
\end{tabular}

NOTE: Because of rounding, percentages may not equal 100.

Abbreviation: AIDS, acquired immune deficiency syndrome.

* For all comparisons, differences are significant at $P<0.01$ using the chi-square test.

${ }^{\dagger}$ The original Medstat disease staging system comprised 5 levels. Due to the small number of patients in levels 1,2 , and 3 , we collapsed these into a single level and named it as level 1 ; we subsequently renamed levels 4 and 5 as levels 2 and 3 , respectively. These levels correspond with the severity of the principal diagnosis, with higher levels indicating more severe disease on admission.

\section{Cost per Episode}

For all 3 conditions, the uninsured had significantly lower age-standardized and sex-standardized costs compared with the privately insured (Table 3). However, Medicaid patients had higher costs than the privately insured for all three 


\begin{tabular}{|c|c|c|c|}
\hline & Privately Insured & Uninsured & Medicaid \\
\hline \multicolumn{4}{|c|}{ In-hospital mortality, rate per 100 discharges (SE) } \\
\hline Acute myocardial infarction & $2.22(0.10)$ & $4.03(0.31)^{*}$ & $4.57(0.34)^{*}$ \\
\hline Stroke & $7.49(0.27)$ & $10.46(0.64)^{*}$ & $9.89(0.45)^{*}$ \\
\hline Pneumonia & $1.75(0.09)$ & $1.74(0.18)$ & $2.48(0.14)^{*}$ \\
\hline \multicolumn{4}{|l|}{ Length of stay, mean (SE), days } \\
\hline Acute myocardial infarction & $4.17(0.06)$ & $4.46(0.09)^{\dagger}$ & $5.85(0.16)^{\dagger}$ \\
\hline Stroke & $6.37(0.13)$ & $7.15(0.25)^{\dagger}$ & $9.28(0.30)^{\dagger}$ \\
\hline Pneumonia & $4.89(0.05)$ & $4.64(0.10)^{\dagger}$ & $5.80(0.08)^{\dagger}$ \\
\hline \multicolumn{4}{|c|}{ Cost per episode, mean (SE), dollars } \\
\hline Acute myocardial infarction & 21,077 (512) & $19,977(833)^{\dagger}$ & 22,452 (841) \\
\hline Stroke & $16,022(679)$ & $14,571(1,036)^{\dagger}$ & $18,462(824)^{\dagger}$ \\
\hline Pneumonia & 8,223 (192) & $7,086(293)^{\dagger}$ & $9,479(271)^{\dagger}$ \\
\hline \multicolumn{4}{|c|}{$\begin{array}{l}\text { NOTE: Age-standardized and sex-standardized using the age and sex distribution of the entire cohort for direct standardization. These are unadjusted } \\
\text { figures. }\end{array}$} \\
\hline \multicolumn{4}{|c|}{ Abbreviations: SE, standard error. } \\
\hline \multicolumn{4}{|c|}{ * Significantly different from privately insured at $P<0.001$ using the chi-square test. } \\
\hline \multicolumn{4}{|c|}{ Significantly different from privately insured at $P<0.05$ using the t-test; log transformations were used to approximate normal distribution. } \\
\hline
\end{tabular}

conditions, significantly so among patients with stroke and pneumonia.

These insurance-related disparities in costs persisted in multivariable analyses (Table 4). The uninsured continued to have lower costs compared with the privately insured, significantly so for patients with AMI and pneumonia. Among stroke and pneumonia patients, Medicaid recipients continued to accrue higher costs than the uninsured or privately insured.

\section{Discussion}

In this nationally representative study of working-age Americans hospitalized for 3 common medical conditions, we found that insurance status was associated with significant variations in in-hospital mortality and resource use. Whereas privately insured patients experienced comparatively lower in-hospital mortality in most cases, mortality risk was highest among the uninsured for 2 of the $3 \mathrm{com}$ mon causes of noncancer inpatient deaths. Although previous studies have examined insurance-related disparities in inpatient care for individual diagnoses and specific populations, no broad overview of this important issue has been published in the past decade. In light of the current economic recession and national healthcare debate, these findings may be a prescient indication of a widening insurance gap in the quality of hospital care.

There are several potential mechanisms for these disparities. For instance, Hadley et al. ${ }^{9}$ reported significant underuse of high-cost or high-discretion procedures among the uninsured in their analysis of a nationally representative sample of 592,598 hospitalized patients. Similarly, Burstin et al. ${ }^{10}$ found that among a population of 30,195 hospital- ized patients with diverse diagnoses, the uninsured were at greater risk for receiving substandard care regardless of hospital characteristics. These, and other similar findings, ${ }^{7,8,19}$ are suggestive of differences in the way uninsured patients are generally managed in the hospital that may partly explain the disparities reported herein.

More specifically, analyses of national registries of AMI have documented lower rates of utilization of invasive, potentially life-saving, cardiac interventions among the uninsured. ${ }^{16,17}$ Similarly, a lower rate of carotid endarterectomy was reported among uninsured stroke patients from an analysis of the 2002 NIS. ${ }^{15}$ Other differences in inpatient management unmeasured by administrative data, such as the use of subspecialists and allied health professionals, may also contribute. ${ }^{32}$ Unfortunately, limitations in the available data prevented us from being able to appropriately address the important issue of insurance related differences in the utilization of specific inpatient procedures.

These disparities may also be indicative of differences in severity of illness that are not captured fully by the MedStat disease staging criteria. The uninsured might have more severe illness at admission, either due to the presence of more advanced chronic disease or delay in seeking care for the acute episode. AMI and stroke are usually the culmination of longstanding atherosclerosis that is amenable to improvement through timely and consistent risk-factor modification. Not having a usual source of medical care, ${ }^{6,33}$ inadequate screening and management of known risk-factors, ${ }^{3,34}$ and difficulties in obtaining specialty care $^{5}$ among the uninsured likely increases their risk of being hospitalized with more advanced disease. The higher likelihood of being admitted through the emergency department ${ }^{19}$ and on weekends ${ }^{9}$ among the uninsured lends credence to the possibility of delays in seeking 


\begin{tabular}{|c|c|c|c|}
\hline & Model $1^{*}$ & Model $2^{\dagger}$ & Model $3^{\ddagger}$ \\
\hline \multicolumn{4}{|c|}{ Acute Myocardial Infarction } \\
\hline Medicaid vs. privately insured & $1.83(1.54-2.18)$ & $1.22(0.99-1.50)$ & $1.15(0.94-1.42)$ \\
\hline \multicolumn{4}{|l|}{ Stroke } \\
\hline Uninsured vs. privately insured & $1.56(1.35-1.80)$ & $1.50(1.30-1.73)$ & $1.49(1.29-1.72)$ \\
\hline Uninsured vs. privately insured & $0.99(0.81-1.21)$ & $1.12(0.91-1.39)$ & $1.10(0.89-1.36)$ \\
\hline Medicaid vs. privately insured & $1.41(1.20-1.65)$ & $1.24(1.04-1.48)$ & $1.21(1.01-1.45)$ \\
\hline \multicolumn{4}{|l|}{ Length of stay, adjusted ratio $\left.(95 \% \mathrm{CI})\right|^{\S}$} \\
\hline \multicolumn{4}{|l|}{ Acute Myocardial Infarction } \\
\hline Uninsured vs. privately insured & $1.00(0.98-1.02)$ & $1.00(0.98-1.02)$ & $1.00(0.98-1.02)$ \\
\hline Medicaid vs. privately insured & $1.17(1.14-1.21)$ & $1.07(1.05-1.09)$ & $1.07(1.05-1.09)$ \\
\hline Medicaid vs. privately insured & $1.15(1.13-1.17)$ & $1.04(1.03-1.06)$ & $1.04(1.03-1.06)$ \\
\hline \multicolumn{4}{|c|}{ Cost per episode, adjusted ratio $(95 \% \mathrm{CI})^{\S}$} \\
\hline \multicolumn{4}{|c|}{ Acute Myocardial Infarction } \\
\hline Uninsured vs. privately insured & $0.97(0.95-0.99)$ & $0.99(0.97-1.00)$ & $0.99(0.97-1.00)$ \\
\hline Medicaid vs. privately insured & $1.01(0.98-1.04)$ & $0.99(0.97-1.01)$ & $0.99(0.97-1.01)$ \\
\hline \multicolumn{4}{|l|}{ Stroke } \\
\hline Uninsured vs. privately insured & $0.97(0.93-1.02)$ & $1.00(0.96-1.03)$ & $1.00(0.97-1.03)$ \\
\hline Medicaid vs. privately insured & $1.17(1.13-1.21)$ & $1.06(1.04-1.09)$ & $1.06(1.04-1.09)$ \\
\hline \multicolumn{4}{|l|}{ Pneumonia } \\
\hline Uninsured vs. privately insured & $0.95(0.92-0.97)$ & $0.98(0.96-1.00)$ & $0.98(0.96-1.00)$ \\
\hline Medicaid vs. privately insured & $1.17(1.15-1.19)$ & $1.05(1.04-1.07)$ & $1.05(1.04-1.07)$ \\
\hline \multicolumn{4}{|c|}{ NOTE: Using multivariable logistic regression models for in-hospital mortality and } \\
\hline
\end{tabular}

care. All of these are potential mediators of higher AMI and stroke mortality in uninsured patients.

Finally, these mortality differences could also be due to the additional risks imposed by poorly managed comorbidities among uninsured patients. Although we controlled for the presence of comorbidities in our analysis, we lacked data about the severity of individual comorbidities. A recent study reported significant lapses in follow-up care after the onset of a chronic condition in uninsured individuals under 65 years of age. ${ }^{34}$ Other studies have also documented insurance related disparities in the care of chronic diseases ${ }^{3,35}$ that were among the most common comorbidities in our cohort.

Most of the reasons for insurance-related disparities noted above for the uninsured are also applicable to Medicaid patients. Differences in the intensity of inpatient care, ${ }^{7,8,15-19}$ limited access to health care services, ${ }^{2,14}$ unmet health needs, ${ }^{5}$ and suboptimal management of chronic medical conditions ${ }^{35}$ were also reported for Medicaid patients in prior research. These factors likely contributed to the higher in-hospital mortality in this patient population, evidenced by the sequential decrease in odds after adjusting for comorbidities and disease severity. Medicaid patients hospitalized for stroke were noted to have significantly longer LOS, which could plausibly be due to difficulties with arranging appropriate discharge disposition; the higher likelihood of paralysis among these patients ${ }^{15}$ would likely necessitate a higher frequency of rehabilitation facility placement. The higher costs for Medicaid patients with stroke and pneumonia may potentially be the result of these patients longer LOS. Although cost differences between the uninsured and privately insured were 
statistically significant, these were not large enough to be of material significance.

\section{Limitations}

Our study has several limitations. Since the NIS does not assign unique patient identifiers that would permit tracking of readmissions, we excluded patients transferred to another acute-care hospital from our study to avoid counting the same patient twice. However, only $10 \%$ of hospitalized patients underwent transfer for cardiac procedures in the National Registry of Myocardial Infarction, with privately insured patients more likely to be transferred than other insurance groups. ${ }^{17}$ Since these patients are also more likely to have better survival, their exclusion likely biased our study toward the null. The same is probable for stroke patients as well.

Some uninsured patients begin Medicaid coverage during hospitalization and should ideally be counted as uninsured but were included under Medicaid in our analysis. They are also likely to be state- and plan-specific variations in Medicaid and private payer coverage that we could not incorporate into our analysis. In addition, we were unable to include deaths that may have occurred shortly after discharge, even though these may have been related to the quality of hospital care. Furthermore, although the 3 conditions we studied are common and responsible for a large number of hospital deaths, they make up about $8 \%$ of total annual hospital discharges, ${ }^{23}$ and caution should be exercised in generalizing our findings to the full spectrum of hospitalizations. Lastly, it is possible that unmeasured confounding could be responsible for the observed associations. Uninsured and Medicaid patients are likely to have more severe disease, which may not be adequately captured by the administrative data available in the NIS. If so, this would explain the mortality association rather than insurance status. $^{36,37}$

\section{Conclusions}

Significant insurance-related differences in mortality exist for 2 of the leading causes of noncancer inpatient deaths among working-age Americans. Further studies are needed to determine whether provider sensitivity to insurance status or unmeasured sociodemographic and clinical prognostic factors are responsible for these disparities. Policy makers, hospital administrators, and physicians should be cognizant of these disparities and consider policies to address potential insurance related gaps in the quality of inpatient care.

\section{Address for correspondence and reprint requests:}

LeRoi S. Hicks, MD, MPH, Division of General Medicine, Brigham and Women's Hospital, 1620 Tremont Street, 3rd Floor, Boston, MA 02120-1613; Telephone: 617-732-5500 ext. 32537; Fax: 617-732-7072; E-mail: lhicks1@partners.org Received 20 March 2009; revision received 21 November 2009; accepted 30 January 2010.

\section{References}

1. Holahan J, Cook A. The U.S. economy and changes in health insurance coverage, 2000-2006. Health Aff (Millwood). 2008;27(2):w135-w144.
2. Weissman JS, Gatsonis C, Epstein AM. Rates of avoidable hospitalization by insurance status in Massachusetts and Maryland. JAMA. 1992;268(17): 2388-2394.

3. Ayanian JZ, Weissman JS, Schneider EC, et al. Unmet health needs of uninsured adults in the United States. JAMA. 2000;284(16):2061-2069.

4. Baker DW, Shapiro MF, Schur CL. Health insurance and access to care for symptomatic conditions. Arch Intern Med. 2000;160(9):1269-1274.

5. Cook NL, Hicks LS, O'Malley AJ, et al. Access to specialty care and medical services in community health centers. Health Aff (Millwood). 2007; 26(5):1459-1468.

6. Wilper AP, Woolhandler S, Lasser KE, et al. A national study of chronic disease prevalence and access to care in uninsured U.S. adults. Ann Intern Med. 2008;149:170-176.

7. Yergan J, Flood AB, Diehr P, LoGerfo JP. Relationship between patient source of payment and the intensity of hospital services. Med Care. 1988; 26(11):1111-1114.

8. Wenneker MB, Weissman JS, Epstein AM. The association of payer with utilization of cardiac procedures in Massachusetts. JAMA. 1990;264(10): 1255-1260.

9. Hadley J, Steinberg EP, Feder J. Comparison of uninsured and privately insured hospital patients: condition on admission, resource use, and outcome. JAMA. 1991;265:374-379.

10. Burstin HR, Lipsitz SR, Brennan TA. Socioeconomic status and risk for substandard medical care. JAMA. 1992;268(17):2383-2387.

11. Ayanian JZ, Kohler BA, Abe T, Epstein AM. The relation between health insurance coverage and clinical outcomes among women with breast cancer. N Engl J Med. 1993;329(5):326-331.

12. Franks P, Clancy CM, Gold MR. Health insurance and mortality. Evidence from a national cohort. JAMA. 1993;270(6):737-741.

13. Sorlie PD, Johnson NJ, Backlund E, Bradham DD. Mortality in the uninsured compared with that in persons with public and private health insurance. Arch Intern Med. 1994;154(21):2409-2416.

14. Cohen JW. Medicaid policy and the substitution of hospital outpatient care for physician care. Health Serv Res. 1989;24:33-66.

15. Shen JJ, Washington EL. Disparities in outcomes among patients with stroke associated with insurance status. Stroke. 2007;38(3):10101016.

16. Sada MJ, French WJ, Carlisle DM, et al. Influence of payor on use of invasive cardiac procedures and patient outcome after myocardial infarction in the United States. Participants in the National Registry of Myocardial Infarction. J Am Coll Cardiol. 1998;31(7):1474-1480.

17. Canto JG, Rogers WJ, French WJ, et al. Payer status and the utilization of hospital resources in acute myocardial infarction: a report from the National Registry of Myocardial Infarction 2. Arch Intern Med. 2000; 160(6):817-823.

18. Calvin JE, Roe MT, Chen AY, et al. Insurance coverage and care of patients with non-ST-segment elevation acute coronary syndromes. Ann Intern Med. 2006;145(10):739-748.

19. Bradbury RC, Golec JH, Steen PM. Comparing uninsured and privately insured hospital patients: Admission severity, health outcomes and resource use. Health Serv Manage Res. 2001;14(3):203-210.

20. Healthcare Cost and Utilization Project. Introduction to the HCUP Nationwide Inpatient Sample (NIS) 2005. Rockville, MD: Agency for Healthcare Research and Quality; 2007:6. Available at: www.hcupus.ahrq.gov/db/nation/nis/NIS_Introduction_2005.pdf. Accessed February 2010 .

21. Healthcare Cost and Utilization Project. Design of the Nationwide Inpatient Sample (NIS) 2005. Rockville, MD: Agency for Healthcare Research and Quality; 2007. Available at: www.hcup-us.ahrq.gov/db/nation/nis/ reports/NIS_2005_Design_Report.pdf. Accessed February 2010.

22. AHRQ Quality Indicators. Inpatient Quality Indicators: Technical Specifications. Version 3.1 (March 12, 2007). Available at: www.qualityindicators.ahrq.gov/downloads/iqi/iqi_technical_specs_v31.pdf. Accessed February 2010.

23. DeFrances CJ, Cullen KA, Kozak LJ. National Hospital Discharge Survey: 2005 annual summary with detailed diagnosis and procedure data. Washington, DC: National Center for Health Statistics; 2007. Vital and Health 
Statistics 13(165). Available at: www.cdc.gov/nchs/data/series/sr_13/ sr13_165.pdf. Accessed February 2010.

24. AHRQ Quality Indicators. Guide to Inpatient Quality Indicators: Quality of Care in Hospitals-Volume, Mortality, and Utilization. Version 3.1 (March 12, 2007). Available at: www.qualityindicators.ahrq.gov/downloads/iqi/iqi_guide_v31.pdf. Accessed February 2010.

25. DeNavas-Walt C, Proctor BD, Smith J. Income, poverty, and health insurance coverage in the United States: 2006. Washington, DC: US Census Bureau. Current Population Reports; 2007: 60-233.

26. Elixhauser A, Russo CA. Conditions Related to Uninsured Hospitalizations, 2003. HCUP Statistical Brief \#8. Rockville, MD: Agency for Healthcare Research and Quality; 2006:6. Available at: www.hcup-us.ahrq.gov/ reports/statbriefs/sb8.pdf. Accessed February 2010.

27. Healthcare Cost and Utilization Project. NIS Description of Data Elements. Available at: www.hcup-us.ahrq.gov/db/nation/nis/nisdde.jsp. Accessed February 2010.

28. Elixhauser A, Steiner C, Harris DR, Coffey RM. Comorbidity measures for use with administrative data. Med Care. 1998;36:8-27.

29. SUDAAN User's Manual, Release 9.0. Research Triangle Park, NC: Research Triangle Institute; 2006.

30. Houchens R, Elixhauser A. Final Report on Calculating Nationwide Inpatient Sample (NIS) Variances, 2001. HCUP Methods Series Report \#2003-2. Online June 2005 (revised June 6, 2005). U.S. Agency for Healthcare Research and Quality. Available at: www.hcup-us.ahrq.gov/
reports/CalculatingNISVariances200106092005.pdf. Accessed February 2010.

31. Binder DA. On the variances of asymptotically normal estimators from complex surveys. Int Stat Rev. 1983;51:279-292.

32. Auerbach AD, Hamel MB, Califf RM, et al. Patient characteristics associated with care by a cardiologist among adults hospitalized with severe congestive heart failure. J Am Coll Cardiol. 2000;36:2119-2125.

33. Pleis JR, Lethbridge-Çejku M. Summary health statistics for U.S. adults: National Health Interview Survey, 2006. Washington, DC: National Center for Health Statistics; 2007:12. Vital and Health Statistics 10(235). Available at: www.cdc.gov/nchs/data/series/sr_10/sr10_235.pdf. Accessed February 2010 .

34. Hadley J. Insurance coverage, medical care use, and short-term health changes following an unintentional injury or the onset of a chronic condition. JAMA. 2007;297(10):1073-1084.

35. Hicks LS, O'Malley AJ, Lieu TA, et al. The quality of chronic disease care in U.S. community health centers. Health Aff (Millwood). 2006;25(6): 1712-1723.

36. Escobar GJ, Greene JD, Scheirer P, Gardner MN, Draper D, Kipnis P. Riskadjusting hospital inpatient mortality using automated inpatient, outpatient, and laboratory databases. Med Care. 2008;46(3):232-239.

37. van Walraven C, Escobar GJ, Greene JD, Forster AJ. The Kaiser Permanente inpatient risk adjustment methodology was valid in an external patient population. J Clin Epidemiol. [E-pub ahead of print]. 